\title{
Degradation intermediates as an indicator of mRNA and cell metabolism
}

\author{
Yusheng Liu $^{2}$, Yiwei Zhang ${ }^{1,2}, \mathrm{Hu} \mathrm{Nie}^{2,3}$, Falong $\mathrm{Lu}^{2,3}$, and Jiaqiang Wang ${ }^{凶}$
}

${ }^{1}$ College of Life Science, Northeast Agricultural University, Harbin 150030, China.

${ }^{2}$ State Key Laboratory of Molecular Developmental Biology, Institute of Genetics and

Developmental Biology, Innovative Academy of Seed Design, Chinese Academy of

Sciences, Beijing 100101, China.

${ }^{3}$ University of Chinese Academy of Sciences, Beijing 100049, China.

凹e-mail: liuys1126@foxmail.com; fllu@genetics.ac.cn; wangjiaqiang@neau.edu.cn; 


\begin{abstract}
Traditional mRNA degradation rate measurements involves complex experimental design with RNA labeling or transcription blocking together with sampling at multiple timepoints $^{1,2}$. These experimental requirements limit the application of transcriptome-wide mRNA degradation rate analysis mainly in cultured cells, but rarely in in vivo samples. Therefore, a direct and simple strategy needs to be developed to study mRNA degradation rate. Here, we defined mRNA degradation intermediates as transcripts where decay is about to occur or has partially occurred in the $3^{\prime}$-untranslated regions after poly(A) tail deadenylation, and found that the proportion of mRNA degradation intermediates is a very simple and convenient indicator for evaluating the degradation rate of mRNA in mouse and human cell lines. In addition, we showed that a higher proportion of mRNA degradation intermediates is correlated with faster cell cycle and higher turnover rate of mouse tissues. Further, we validated that in mouse maturing oocytes where transcription is silent ${ }^{3,4}$, the proportion of mRNA degradation intermediates is positively correlated with the mRNA degradation rate. Together, these results demonstrate that degradation intermediates can function as a good indicator of mRNA, cell, and tissue metabolism, and can be easily assayed by total RNA 3'-end sequencing from a single bulk cell sample without the need for drug treatment or multi-timepoint sampling. This finding is of great potential for studies on mRNA degradation rate at the molecular, cellular, or organic level, including samples or systems that cannot be assayed with previous methods. In addition, further application of the findings into single cells will likely greatly aid the identification and study of rare cells with unique cellular metabolism dynamics such as tissue stem cells and tumor cells.
\end{abstract}




\section{Introduction}

As the bridge between genes and functional proteins, mRNA metabolism is crucial for the control of gene expression, including synthesis, and degradation. The individual biochemical steps of mRNA degradation have been well studied ${ }^{1,2}$. Half-life has been considered a good indicator of mRNA degradation rate ${ }^{5,6}$, because mRNA degradation profiles typically match a simple exponential decay. There are two main approaches to study the mRNA half-lives at the transcriptome scale. The first approach involves global inhibition of transcription with drug or genetic perturbation, and the half-life can then be determined by the loss of mRNA over time since inhibition ${ }^{6-8}$. The second approach involves metabolic labelling, and the half-life can be determined from either the disappearance of the mRNA or the kinetics of initial labelling ${ }^{1,2,9,10}$. However, both approaches require perturbation of transcription, introducing potential bias in quantification of mRNA half-life. In addition, both methods require a large number of cells for analysis over successive time points, limiting the use to model cell lines that can be easily obtained in bulk and accessible to the exogenous treatments. Furthermore, different methods have been shown to yield very different ranges of mRNA half-life estimation for the same cultured cell lines, with a relatively low correlation between the measured halflives $^{5,9,11}$. Thus, it is very challenging to accurately analyze mRNA metabolism in a noncell line system, such as in tumor migration and embryonic organogenesis.

With few exceptions, mRNA degradation begins with the deadenylation-linked removal of the RNA poly(A) tail ${ }^{2,12-14}$, which is one of the essential structural components of mRNA $^{15-17}$. Thus, in a population of unsynchronized cells for each gene there must be a stable proportion of mRNA degradation intermediates - transcripts where decay is about to occur or has partially occurred in the 3 '-untranslated region (UTR) after poly(A) tail deadenylation, because each point of the mRNA metabolism wave has been covered by the large number of unsynchronized cells. This raises the question of whether the proportion of mRNA degradation intermediates reflect the degradation rate of mRNAs. 
Here, we found that different genes indeed have different proportion of degradation intermediates among their mRNA transcripts in mouse NIH 3 T3 cells (3T3), mouse embryonic stem cells (mES) and human Hela cells (Hela). Additionally, we found a strong correlation between the proportion of mRNA degradation intermediates and the mRNA degradation rate in both unsynchronized bulk cells and synchronized maturing oocytes. These findings suggest that mRNA degradation intermediates can be used as a simple indicator to measure mRNA degradation rate at the transcriptome scale in any samples that can undergo 3'-adaptor ligation-based high-throughput RNA sequencing, such as PAIsoseq 2 and TAIL-seq ${ }^{18,19}$

\section{Results}

\section{Mathematic model of mRNA degradation rate and the proportion of degradation intermediates}

Typical mRNA molecules are transcribed in the nucleus and polyadenylated immediately following transcription. The poly(A)-tailed mRNAs are then exported to the cytoplasm for translation. For a single mRNA molecule life cycle, there is an adenylated stable transcript phase followed by a deadenylated degradation intermediate phase. It has been known for thirty years that, with few exceptions, bulk mRNA undergo decay initiation by poly(A)-tail shortening ${ }^{2,12-14}$. After poly(A) tail removal, the unprotected 3' end is attacked and digested by a $3^{\prime}-5^{\prime}$ exonuclease ${ }^{16}$. Therefore, mRNA degradation intermediates, meaning transcripts where decay is about to occur or has partially occurred in the $3^{\prime}-\mathrm{UTR}$ after poly(A) tail deadenylation, exist widely in cells (Fig. 1a).

In a single cell, mRNA molecules are transcribed, processed, and degraded following an inherent procedure, forming a cell type-specific mRNA metabolism wave as the cell cycle progresses (Fig. 1b, top). Thus, only continuous time points that cover at least one mRNA metabolism wave, but not any single time point, will give an accurate map of mRNA decay for a single cell (Fig. 1b, top). For bulk cells of the same type, in contrast, there are enough number of cells covering any point of the mRNA metabolism wave and any point of the 
cell cycle at a single time point (Fig. 1b, bottom). Because the proportion of mRNA degradation intermediates can be easily generated from data of any bulk samples, we hypothesize that there should be mathematical models that can 1) depict the whole mRNA metabolism wave of one bulk sample based on data from a single time point, and 2) correlate the proportion of mRNA degradation intermediates with mRNA degradation rate.

Because decay for each transcript typically matches a simple exponential decay function, we built a model using different exponential decay functions with different base numbers, and normalized them with a same initial length (Fig. 1c; $y=C$ when $\mathrm{x}=0$ ). We found that the proportion of mRNA degradation intermediates (under the blue dotted line in which the tail has been fully deadenylated) is positively correlated with the base number of exponential decay functions, reflecting the principle that the higher the metabolic rate is, the higher the proportion of mRNA degradation intermediates will be (Fig. 1c). In other words, the longer the mRNA half-life is, the lower the proportion of mRNA degradation intermediates will be.

In order to test our mathematical model, we analyzed half-life data for several representative genes determined by 5-ethynyl uridine (5EU) metabolic labelling ${ }^{1}$ and calculated the proportion of mRNA degradation intermediates from our PAIso-seq 2 data of $3 \mathrm{~T} 3^{19}$. There was a negative correlation between half-life and the proportion of mRNA degradation intermediates (Fig. 1d), indicating an increasing proportion of mRNA degradation intermediates along with faster mRNA degradation, which fits our mathematical model. Therefore, we propose that in bulk cells, the proportion of mRNA degradation intermediates is positively correlated with mRNA degradation rate and negatively correlated with mRNA half-life.

\section{Proportion of mRNA degradation intermediates negatively correlates with mRNA half-life}

With our PAIso-seq2 dataset for $3 \mathrm{~T} 3$ and $\mathrm{mES}^{19}$, we calculated the transcriptome-wide 
proportion of mRNA degradation intermediates and compared it with the mRNA half-life data from prior studies ${ }^{1,7,8,10}$. We collected three mRNA half-life datasets of 3T3 cells: two from multi-time point 5EU metabolic labelling ${ }^{1}$, and the other from single time-point 4thiouridine $(4 \mathrm{~s} U)$ metabolic labelling ${ }^{10}$. Strong negative correlations $(\mathrm{Rp}=-0.57$ to -0.44$)$ were observed between the proportion of mRNA degradation intermediates and mRNA half-life in all three datasets (Fig. 2a). We also collected three mRNA half-life datasets of mES, all of which use global transcription inhibition by RNA polymerase inhibitor treatment $^{7,8}$. As a result, moderate negative correlations $(\mathrm{Rp}=-0.37$ to -0.25$)$ between the proportion of mRNA degradation intermediates and mRNA half-life were also observed in mES (Fig. 2b).

In addition to the PAIso-seq2 data, we analyzed a published TAIL-seq dataset for $\mathrm{HeLa}^{20,21}$, and found that the proportion of mRNA degradation intermediates calculated from TAIL-seq data ${ }^{22}$ showed a negative correlation $(\mathrm{Rp}=-0.36$ to -0.34$)$ with mRNA halflife in $\mathrm{HeLa}^{20,21}$ (Fig. 2c). Taken together, these transcriptome-wide analyses reveal that the proportion of mRNA degradation intermediates negatively correlates with mRNA halflife.

\section{Proportion of mRNA degradation intermediates behaves well to indicate cell cycle duration}

The RNA metabolism wave is associated with the cell cycle ${ }^{23}$. In addition, the average/median mRNA half-life is known to be associated with cell cycle duration. For example, the mRNA degradation lifetime is roughly five minutes in E. coli, 20 minutes in yeast and 10 hours in human cells; these values are positively correlated with their cell cycle times (roughly 30 minutes for E. coli, 90 minutes for yeast, and 50 hours for human cells). Therefore, we speculated that the global proportion of mRNA degradation intermediates would be elevated in rapidly cycling cells (Fig. 3a). To validate this, we compared the proportion of mRNA degradation intermediates at the transcriptome level in 
the mouse cell lines 3T3 (cell cycle duration: $20-26$ hours [http://www.nih3t3.com/]) and mES (cell cycle duration: $12-17$ hours $^{24}$ ). As expected, the global proportion of mRNA degradation intermediates was significantly higher in mES compared to 3T3 (Fig. 3b). Moreover, the proportion of mRNA degradation intermediates for individual genes in $\mathrm{mES}$ were significantly higher than those in $3 \mathrm{~T} 3$ (Fig. 3c). For example, Aco2, Ybx1, Eeflb2, Slc25a3, Srsf3, and Atrx all showed a higher proportion of mRNA degradation intermediates in mES than in $3 \mathrm{~T} 3$ (Fig. 3d). Together, these results indicate that the proportion of mRNA degradation intermediates negatively correlate with cell cycle length, making it a good indicator of cell cycle duration time.

\section{Proportion of mRNA degradation intermediates works well as indicator of the mouse tissue turnover rate}

To determine whether mRNA degradation can be used to infer the turnover rate of tissues, we calculated the proportion of mRNA degradation intermediates for different mouse tissues $^{19}$, and found that different tissues have different proportions of mRNA degradation intermediates (Fig. 4a). Previous data comparing the turnover rate of mouse muscle to liver, and muscle to heart ${ }^{25,26}$ (Fig. 4b), showed that the absolute turnover rate values are very different. However, the relative turnover rate of these three tissues are clear: the heart took about half the time that muscle took for full turnover, while the liver took less than $30 \%$ of the time of muscle (Fig. 4b). Thus, among these three tissues, muscle turnover is slowest, liver turnover is fastest, and heart turnover is in the middle. Importantly, the proportion of mRNA degradation intermediates at the transcriptome level correlates well with the tissue turnover rate for these three tissues (Fig. 4a). In addition, the proportion of mRNA degradation intermediates for individual genes were also correlated with tissue turnover rates (Fig. 4c). For example, the proportion of mRNA degradation intermediates for Eif4g2, Ube2d3, Gnas, and Cnbp were correlated with the turnover rate of the corresponding tissue (Fig. 4d). Together, these results revealed that the proportion of mRNA degradation 
intermediates is positively correlated with tissue turnover rate and negatively correlated with tissue turnover time, and can be used as an indicator of tissue turnover rate.

\section{Proportion of mRNA degradation intermediates indicates the maternal RNA decay rate during mouse oocyte maturation}

Transcription is silent in mammalian fully grown oocytes at germinal vesicle stage (GV oocytes). Therefore, the oocyte maturation process, ranging from GV stage to MI stage and MII stage, provides an ideal physiological system to analyze mRNA decay without the complication of new transcription occurring. We found that the proportion of mRNA degradation intermediates at the transcriptome level increased during oocyte maturation (Fig. 5a), which is consistent with global degradation of mRNA during this process ${ }^{27}$. Consistently, the proportion of mRNA degradation intermediates at the gene level also increased greatly during this process (Fig. 5b). For most of the genes analyzed, the proportion of mRNA degradation intermediates of individual genes showed an increase between different stages during oocyte maturation (Fig. 5c).

To determine whether the proportion of mRNA degradation intermediates at the current stage can indicate the mRNA degradation rate from the previous stage to the current stage, we built a mathematical model based on exponential decay functions for synchronized maturing oocytes. The proportion of mRNA degradation intermediates was positively correlated with the base number of exponential decay functions, reflecting that higher degradation rate is correlated with a higher proportion of mRNA degradation intermediates (Fig. 5d). To test the mathematical model, we used the ratio of transcripts per million (TPM) of the current stage to the previous stage as the mRNA degradation rate (smaller value indicates higher decay rate), based on which the genes were grouped into four groups (Fig. $5 \mathrm{e})$. The results showed that mRNA degradation rate was positively correlated with the proportion of mRNA degradation intermediates during oocyte maturation (Fig. 5e). Together, these results reveal that mRNA degradation intermediates can indicate RNA 
decay rate in both of cell cycle synchronized cells (like maturating oocyte) and unsynchronized bulk cells.

\section{Discussion}

Gene expression is determined by the equilibration of mRNA transcription, and RNA decay ${ }^{16}$. Traditionally, transcriptome-wide mRNA half-lives are determined by RNA quantification after metabolic labelling or global transcription inhibition ${ }^{1,2}$. All these approaches involve drug treatment or genetic perturbation coupled with multiple sampling at a series of time points. These complicated experiments are technically challenging and often yield very different results between different labs for the same cell lines, such as 3T3 ${ }^{1,7,8,10}$ (Extended Data Fig. 1). In addition, half-lives are generated through statistical modelling, which is prone to variation caused by different signal to noise levels between experiments. Additionally, drugs that disrupt Pol II-driven transcription also affect stress responses, and other pathways. Thus, not only do the average half-lives differ, but the correlation between half-lives measured with these methods is generally very low ${ }^{5}$. Using unsynchronized bulk cells and synchronized maturing oocytes, we here show that the proportion of mRNA degradation intermediates is a reliable indicator of mRNA degradation rate (Fig. 6). This new indicator has the following advantages: no requirement for drug or genetic treatment; digitalized readout directly from counts, directly comparable between samples and likely between data generated from different labs; and just need a single time point, highly sensitive assay, which enables analysis of low-input samples.

Different cell lines and tissues have different proportions of mRNA degradation intermediates, and higher mRNA metabolism rate of a cell line or tissue corresponds to a higher proportion of mRNA degradation intermediates. In addition, the proportion of mRNA degradation intermediates of the same gene in different tissues also differs. These findings suggest that the proportion of mRNA degradation intermediates is of the potential to distinguish between different cell types. With the continuous progress of single cell 
technology, more detailed cell typing and functional research are needed. Single-cell mRNA degradation rate measurement maybe can be one of the important indicators for cell typing in the future. However, the current high-throughput single-cell RNA sequencing methods all rely on poly(A) tails to capture mRNAs, which are restricted to polyadenylated mRNA molecules and are completely unable to capture the degradation intermediates. Additionally, current methods cannot evaluate the mRNA degradation rate at the single cell level. Thus, a new single-cell RNA sequencing technology that can sequence the 3 '-ends of total RNA regardless of the polyadenylation status (excluding rRNA) would be suitable for mRNA degradation rate evaluation at the single cell level, which will also help to define cell types more accurately.

mRNA metabolism and cell cycle are faster in cancer cells than in normal cells. Thus, we speculate that cancer cells would contain higher proportion of mRNA degradation intermediates than normal cells, which would be a novel indicator for cancer screening of great potential in the future. Additionally, mRNA metabolism may be different in adult stem cells, progenitors, and differentiated cells; thus, the proportion of mRNA degradation intermediates may be useful for identification of adult stem cells, which have key roles in tissue regeneration. We envision that the indicator we have identified here will enable study of mRNA metabolism dynamics during embryonic development as well as disease processes.

\section{Materials and Methods}

\section{mRNA degradation intermediates analysis}

PAIso-seq2 sequencing data were processed as described ${ }^{19}$. Clean CCS reads were aligned to Mus musculus UCSCmm10 reference genome using minimap2 (v.217-r941) ${ }^{28}$. Then, poly(A) tail was extracted using python script PolyA_trim.py (http://). The 3'-soft clip sequence of the CCS reads in the alignment file is used as candidate poly(A) tail sequence. 
The 3 '-soft clip sequences with the frequency of U, C and G greater or equal to 0.1 simultaneously were marked as "HIGH_TCG" tails. To better definite the poly(A) tail, we defined a continuous score based on the transitions between the two adjacent nucleotide residues throughout the $3^{\prime}$-soft clip sequences. To calculate continuous score, the transition from one residue to the same residue scored 0 , and the transition from one residue to a different residue score 1 . The 3'-soft clips which were not marked as "HIGH_TCG" and with continuous score less than or equal to 12 were considered as poly(A) tails. Finally, transcripts without poly(A) tail were treated as mRNA degradation intermediates.

\section{Statistical analyses}

Statistical analyses (mean \pm standard error of the mean [SEM]) were performed in Excel. Statistical significance was calculated with Student's t-test.

\section{Data availability}

PAIso-seq2 data for $3 \mathrm{~T} 3$ cells, mES cells, 12 mouse tissues (uterus, liver, brain, brown fat, lung, kidney, spleen, oviduct, intestine, epididymis, white fat, and muscle), and mouse oocytes (GV, MI, and MII stages), have been previously described ${ }^{19,29-31}$. The ccs data in bam format from PAIso-seq1 and PAIso-seq2 experiments will be available at Genome Sequence Archive hosted by National Genomic Data Center. This study includes analysis of the following published data: RNA half-life data of Eisen et al. (Gene Expression Omnibus database (GEO) accession no. GSE134660) used in Fig. 1d and Fig. 2a; Schwanhäusser et al. (Sequence Read Archive database (SRA) accession no. SRA030871) used in Fig. 2a; Ke et al. (GEO accession no. GSE86336) used in Fig. 2b; Sharova et al. (GEO accession no. GSE13609) used in Fig. 2b; Tani et al. (DRASearch accession no. DRA000345, DRA000346, DRA000347, DRA000348, and DRA000350) used in Fig. 2c; Lim et al. (GEO accession no. GSE59628) used in Fig. 2c; and mouse oocyte RNA-seq data of Sha, et al. (GEO accession no. GSE118564) used in Fig. 5a. Custom scripts used for data analysis will be available upon request. 


\section{Acknowledgements}

This work was supported by the National Key Research and Development Program of China (2018YFA0107001), the Strategic Priority Research Program of the Chinese Academy of Sciences (XDA24020203), National Natural Science Foundation of China (31970588, 32170606), Natural Science Foundation of Heilongjiang province (YQ2020C003), the China Postdoctoral Science Foundation (2020M670516, 2020T130687), and the State Key Laboratory of Molecular Developmental Biology.

\section{Author Contributions}

Yusheng Liu, Falong Lu and Jiaqiang Wang conceived the project and designed the study. Yusheng Liu, Yiwei Zhang, Hu Nie, Falong Lu and Jiaqiang Wang analyzed the sequencing data. Yusheng Liu and Jiaqiang Wang organized all figures. Yusheng Liu, Falong Lu and Jiaqiang Wang supervised the project. Yusheng Liu, Falong Lu and Jiaqiang Wang wrote the manuscript with the input from the other authors.

\section{Competing Interests statement}

The authors declare no competing interests.

\section{Reference}

1 Eisen, T. J. et al. The Dynamics of Cytoplasmic mRNA Metabolism. Mol Cell 77, 786-799 e710, doi:10.1016/j.molcel.2019.12.005 (2020).

2 Passos, D. O. \& Parker, R. Analysis of cytoplasmic mRNA decay in Saccharomyces cerevisiae. Methods Enzymol 448, 409-427, doi:10.1016/S0076-6879(08)02620-7 (2008).

3 Liu, Y. et al. BTG4 is a key regulator for maternal mRNA clearance during mouse early embryogenesis. J Mol Cell Biol 8, 366-368, doi:10.1093/jmcb/mjw023 (2016).

$4 \mathrm{Yu}, \mathrm{C}$. et al. BTG4 is a meiotic cell cycle-coupled maternal-zygotic-transition licensing factor in oocytes. Nat Struct Mol Biol 23, 387-394, doi:10.1038/nsmb.3204 (2016).

5 Geisberg, J. V., Moqtaderi, Z., Fan, X., Ozsolak, F. \& Struhl, K. Global analysis of mRNA isoform halflives reveals stabilizing and destabilizing elements in yeast. Cell 156, 812-824, doi:10.1016/j.cell.2013.12.026 (2014).

6 Grigull, J., Mnaimneh, S., Pootoolal, J., Robinson, M. D. \& Hughes, T. R. Genome-wide analysis of mRNA stability using transcription inhibitors and microarrays reveals posttranscriptional control of ribosome biogenesis factors. Mol Cell Biol 24, 5534-5547, doi:10.1128/MCB.24.12.5534-5547.2004 (2004). 
Sharova, L. V. et al. Database for mRNA half-life of 19977 genes obtained by DNA microarray analysis of pluripotent and differentiating mouse embryonic stem cells. DNA Res 16, 45-58, doi:10.1093/dnares/dsn030 (2009).

$\mathrm{Ke}$, S. et al. m(6)A mRNA modifications are deposited in nascent pre-mRNA and are not required for splicing but do specify cytoplasmic turnover. Genes Dev 31, 990-1006, doi:10.1101/gad.301036.117 (2017).

9 Sun, M. et al. Comparative dynamic transcriptome analysis (cDTA) reveals mutual feedback between mRNA synthesis and degradation. Genome Res 22, 1350-1359, doi:10.1101/gr.130161.111 (2012).

10 Schwanhausser, B. et al. Global quantification of mammalian gene expression control. Nature 473, 337-342, doi:10.1038/nature10098 (2011).

11 Baudrimont, A. et al. Multiplexed gene control reveals rapid mRNA turnover. Science advances 3, e1700006, doi:10.1126/sciadv.1700006 (2017).

12 Muhlrad, D. \& Parker, R. Mutations affecting stability and deadenylation of the yeast MFA2 transcript. Genes Dev 6, 2100-2111, doi:10.1101/gad.6.11.2100 (1992).

Shyu, A. B., Belasco, J. G. \& Greenberg, M. E. Two distinct destabilizing elements in the c-fos message trigger deadenylation as a first step in rapid mRNA decay. Genes Dev 5, 221-231, doi:10.1101/gad.5.2.221 (1991).

14 Chan, L. Y., Mugler, C. F., Heinrich, S., Vallotton, P. \& Weis, K. Non-invasive measurement of mRNA decay reveals translation initiation as the major determinant of mRNA stability. elife 7, doi:10.7554/eLife.32536 (2018).

15 Parker, R. RNA degradation in Saccharomyces cerevisae. Genetics 191, 671-702, doi:10.1534/genetics.111.137265 (2012).

16 Garneau, N. L., Wilusz, J. \& Wilusz, C. J. The highways and byways of mRNA decay. Nat Rev Mol Cell Biol 8, 113-126, doi:10.1038/nrm2104 (2007).

17 Balagopal, V., Fluch, L. \& Nissan, T. Ways and means of eukaryotic mRNA decay. Biochim Biophys Acta 1819, 593-603, doi:10.1016/j.bbagrm.2012.01.001 (2012).

Chang, H., Lim, J., Ha, M. \& Kim, V. N. TAIL-seq: genome-wide determination of poly(A) tail length and 3' end modifications. Mol Cell 53, 1044-1052, doi:10.1016/j.molcel.2014.02.007 (2014).

Liu, Y., Nie, H., Zhang, Y., Lu, F. \& Wang, J. Comprehensive analysis of mRNA poly(A) tail reveals complex and conserved regulation. bioRxiv Processing (2021).

Lim, J. et al. Uridylation by TUT4 and TUT7 marks mRNA for degradation. Cell 159, 1365-1376, doi:10.1016/j.cell.2014.10.055 (2014).

21 Tani, H. et al. Genome-wide determination of RNA stability reveals hundreds of short-lived noncoding transcripts in mammals. Genome Res 22, 947-956, doi:10.1101/gr.130559.111 (2012). $\mathrm{Yi}, \mathrm{H}$. et al. PABP Cooperates with the CCR4-NOT Complex to Promote mRNA Deadenylation and Block Precocious Decay. Mol Cell 70, 1081-1088 e1085, doi:10.1016/j.molcel.2018.05.009 (2018). Liu, Y., Nie, H. \& Lu, F. Dynamic RNA 3' Uridylation and Guanylation during Mitosis. iScience 23, 101402, doi:10.1016/j.isci.2020.101402 (2020).

24 Takahashi, K. \& Yamanaka, S. Induction of pluripotent stem cells from mouse embryonic and adult 
fibroblast cultures by defined factors. Cell 126, 663-676, doi:10.1016/j.cell.2006.07.024 (2006).

25 MacAvoy;, S. E., Macko;, S. A. \& Arneson, L. S. Growth versus metabolic tissue replacement in mouse tissues determined by stable carbon and nitrogen isotope analysis. Canadian Journal of Zoology 83, 631-641, doi:DOI: 10.1139/Z05-038 (2005).

26 Fornasiero, E. F. et al. Precisely measured protein lifetimes in the mouse brain reveal differences across tissues and subcellular fractions. Nat Commun 9, 4230, doi:10.1038/s41467-018-06519-0 (2018).

27 Sha, Q. Q. et al. CNOT6L couples the selective degradation of maternal transcripts to meiotic cell cycle progression in mouse oocyte. EMBO J 37, doi:10.15252/embj.201899333 (2018).

28 Li, H. Minimap2: pairwise alignment for nucleotide sequences. Bioinformatics 34, 3094-3100, doi:10.1093/bioinformatics/bty191 (2018).

29 Liu, Y. et al. Abundant non-A residues in the poly(A) tail orchestrate the mouse oocyte-to-embryo transition. bioRxiv Processing (2021).

30 Liu, Y. et al. Poly(A) tail length is a major regulator of maternal gene expression during the mammalian oocyte-to-embryo transition. bioRxiv Processing (2021).

31 Liu, Y. et al. Re-polyadenylation occurs predominantly on maternal mRNA degradation intermediates during mammalian oocyte-to-embryo transition. bioRxiv Processing (2021). 


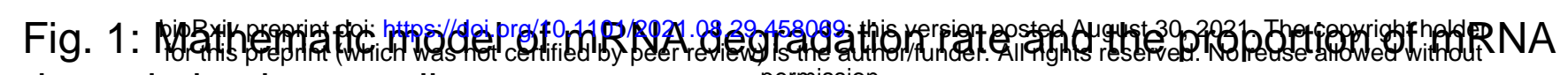
degradation intermediates

a

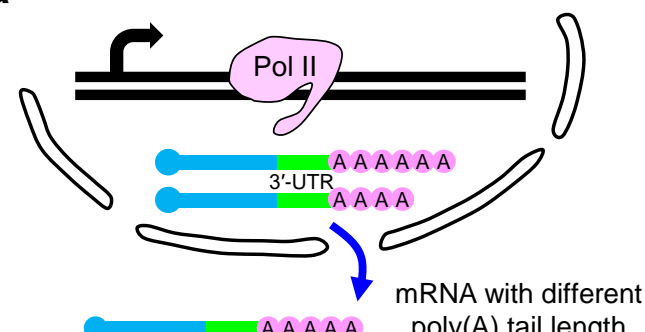
CAA

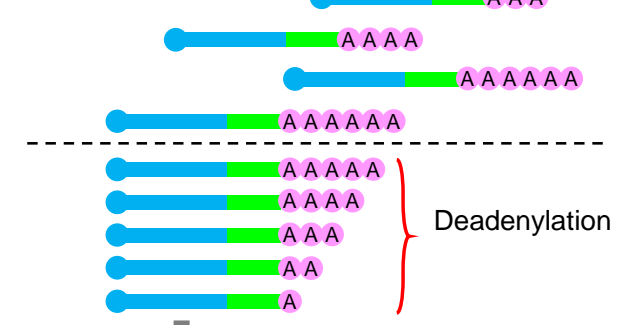

$\sqrt{2}$

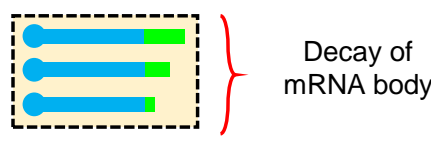

mRNA degradation intermediates b

Single cell: One point in Cell cycle and cannot represent mRNA metabolism process

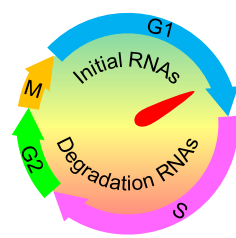

$\bullet \bullet \bullet$

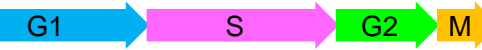

(O) (48)

(O)

mRNA metabolism wave

Bulk cells: Cover Cell cycle at any points and can simulate mRNA metabolism process
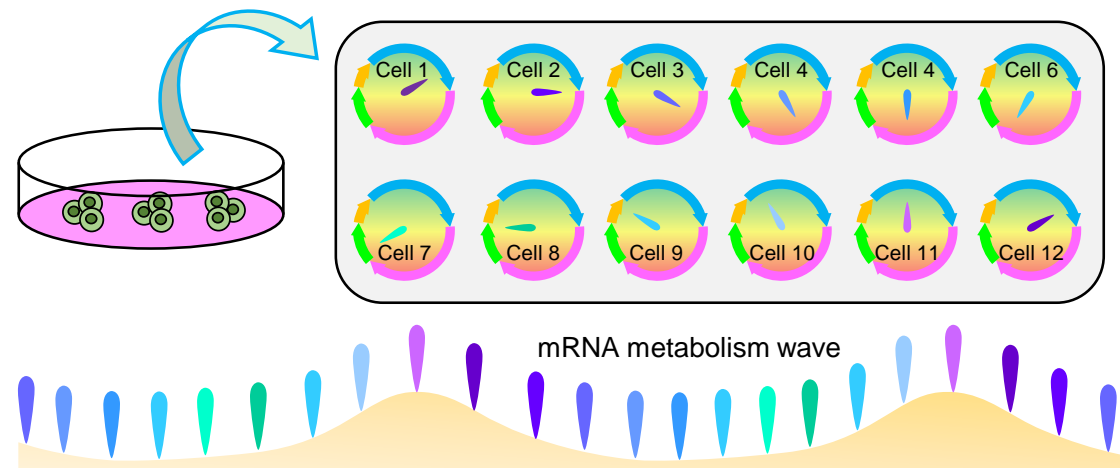

C

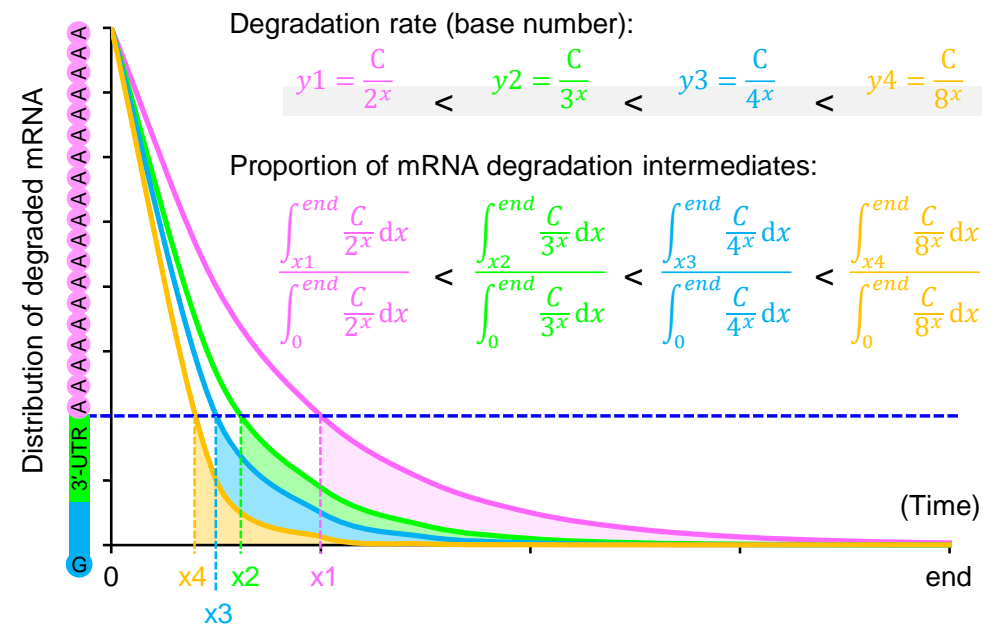

d 3 3т3

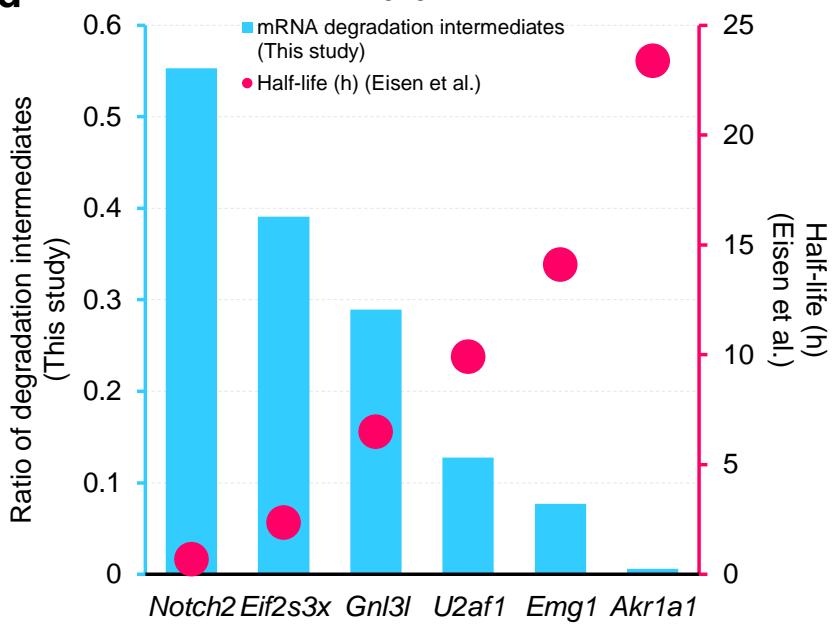




\section{Figure legends}

Fig. 1 | Mathematic model of mRNA metabolism rate and the proportion of mRNA degradation intermediates.

a, Model showing the generation of tail-less mRNA decay intermediates (mRNA degradation intermediates) in cells.

b, Model showing that one time point in cell cycle cannot represents mRNA metabolism process in a single cell, while one time point in cell cycle can simulates mRNA metabolism process in bulk cells.

c, Mathematical model correlating mRNA metabolism rate and the proportion of mRNA degradation intermediates. We put together different exponential decay functions with different base numbers, and normalized them with a same initial length $(\mathrm{y}=\mathrm{C}$ when $\mathrm{x}=$ 0 ). For function y1, the ratio of the degradation intermediates area (under the y1 line, above the $\mathrm{x}$ axis, and right of $\mathrm{x} 1$ ), to the total area (under the $\mathrm{y} 1$ line, the $\mathrm{x}$ axis, and right to the $\mathrm{y}$ axis) represents the proportion of mRNA degradation intermediates. The positive correlation between mRNA metabolism rate and the proportion of mRNA degradation intermediates is shown.

d, Half-lives measured by data from previous studies ${ }^{1}$ and the proportion of mRNA degradation intermediates measured by PAIso-seq2 for Notch2, Eif2s3x, Gnl3l, U2af1, Emgl, and Akrlal of $3 \mathrm{~T} 3$ cells. 
Fig. 2 Proportion of mRNA degradation intermediates negatively correlates with mRNA half-life.

Scatter plot of the proportion of mRNA degradation intermediates and mRNA half-lives at the transcriptome scale in $3 \mathrm{~T}^{1,10}(\mathbf{a}), \mathrm{mES}^{7,8}(\mathbf{b})$, and $\mathrm{HeLa}^{20-22}(\mathbf{c})$ measured by PAIsoseq2. Each dot represents one gene. Genes with at least 10 reads in each sample are included in the analysis. Pearson's correlation coefficient (Rp), Spearman's rank correlation coefficient (Rs), and number of genes included in the analysis are shown on the bottom left of each graph. 


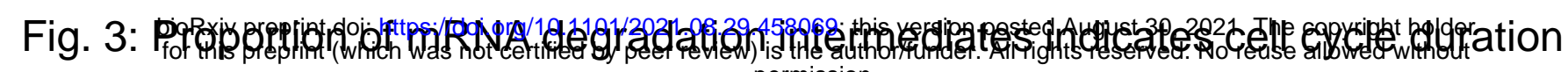
a

Clock Normalized Cell cycle with long

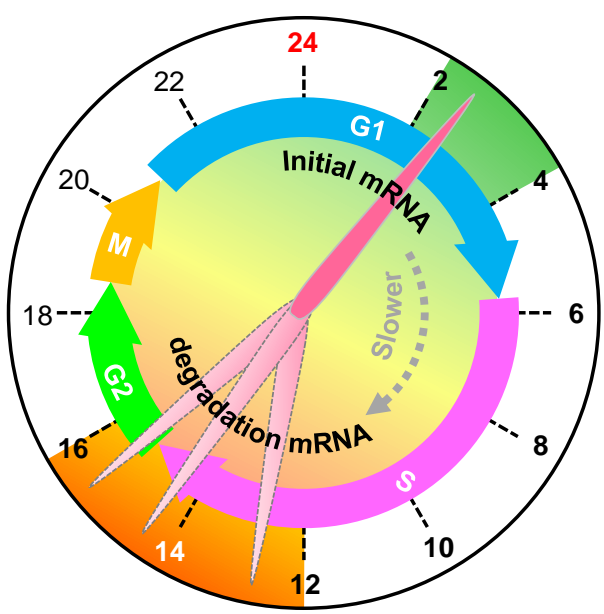

The red and solid line hand points initial mRNA area permission.

Clock Normalized Cell cycle with short

The shorter cell cycle<smiles>c1ccccc1</smiles>

The shorter time of reaching designated area

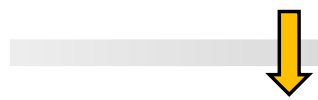

The faster the mRNA metabolism rate<smiles>c1ccccc1</smiles>

The mRNA degradation intermediates appears the more frequently in the designated area at the same time

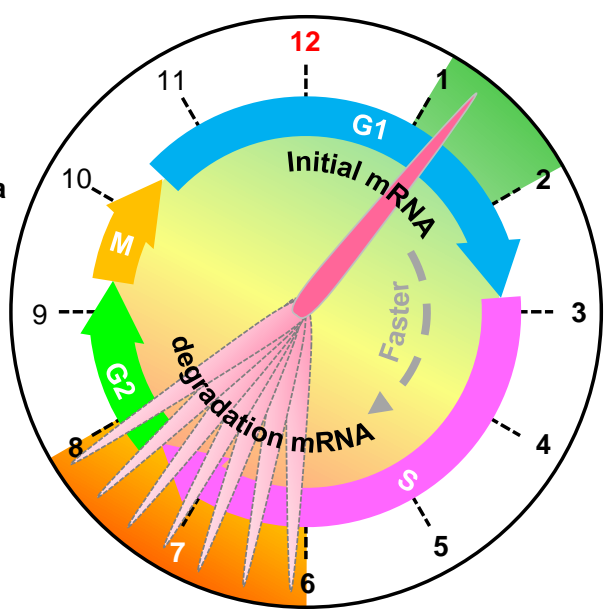

The light red and dotted line hand points mRNA degradation intermediates area

d

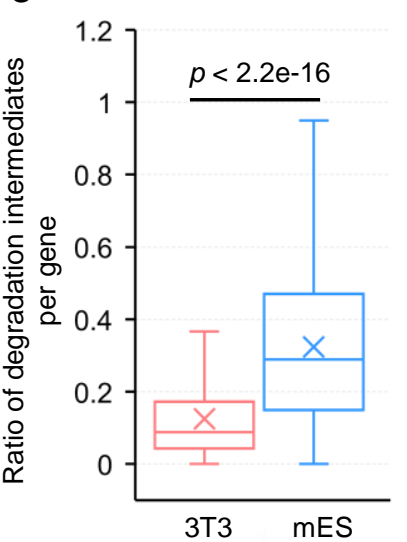

c

mES

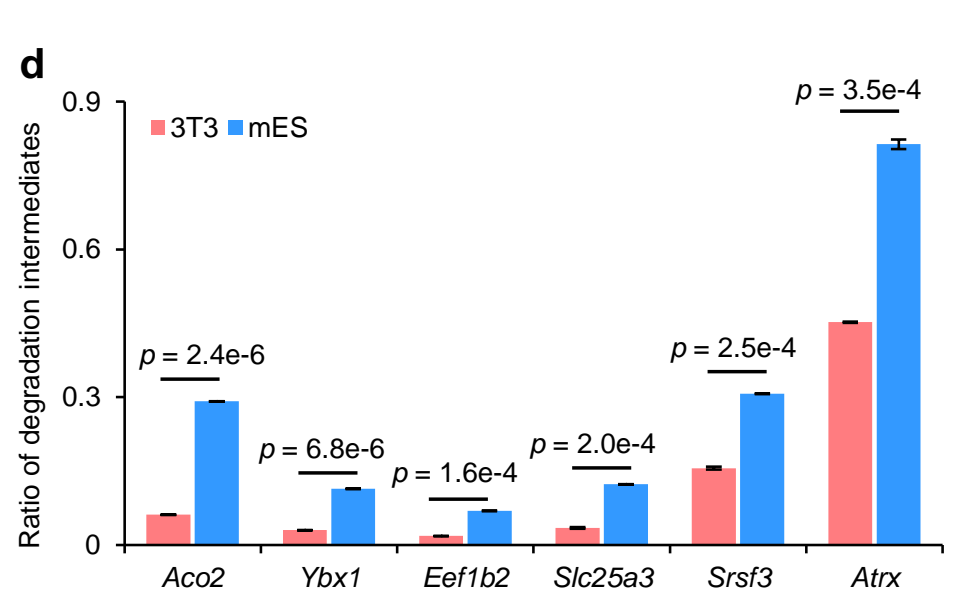

b

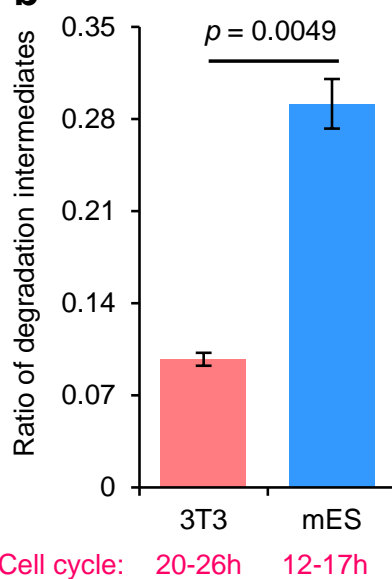




\section{Fig. 3 | Proportion of mRNA degradation intermediates indicates cell cycle duration.}

a, Model showing that the proportion of mRNA degradation intermediates will be elevated in rapid cycling cells. For different cell cycles, the mRNA metabolism wave is associated with the cell cycle, so the proportion of initial (newly transcribed) mRNAs (dark green) and mRNA degradation intermediates (orange) are similar. For a fixed period of time (for example, $72 \mathrm{~h}$ ), the hand points to the mRNA degradation intermediates region fewer times in the slower cell cycle (about three times; left) and more times in the faster cell cycle (about six times; right).

b, Proportion of mRNA degradation intermediates at the transcriptome level in 3T3 and mES measured by PAIso-seq2. Cell cycle durations are shown below the x-axis. Error bars indicate standard error of the mean (SEM) from two replicates. $p$-value was calculated with Student's $t$-test.

c, Box plot of the proportion of mRNA degradation intermediates of individual genes in $3 \mathrm{~T} 3$ and mES measured by PAIso-seq2. Genes with at least 20 reads in the $3 \mathrm{~T} 3$ and mES sample are included in the analysis $(n=4,348)$. The " $x$ " indicates the mean value, the horizontal bars show the median value, and the top and bottom of the box represent the value of 25 th and 75 th percentile, respectively. $p$-value was calculated with Student's $t$-test. d, Proportion of mRNA degradation intermediates of Aco2, Ybx1, Eef1b2, Slc25a3, Srsf3, and Atrx in $3 \mathrm{~T} 3$ and mES measured by PAIso-seq2. Error bars indicate SEM from two replicates. $p$-value was calculated with Student's $t$-test. 


\section{Fig. 4 | Proportion of mRNA degradation intermediates works well as indicator of the} mouse tissue turnover rate.

a, Proportion of mRNA degradation intermediates across 13 mouse tissues measured by PAIso-seq2. Liver is shown in red; heart is shown in green; muscle is shown in blue; other tissues are shown in gray.

b, Previously reported data ${ }^{25,26}$ for the turnover time of mouse muscle, liver, and heart tissues. Muscle turnover is the slowest, whereas liver turnover is the fastest, and heart is in the middle. MS, mass spectrum.

c, Box plot of the proportion of mRNA degradation intermediates of individual genes in mouse liver, heart, and muscle measured by PAIso-seq2. Genes with at least 20 reads in each sample are included in the analysis $(\mathrm{n}=1,351,1,469$, and 1,575 for muscle, liver, heart, respectively). The " $x$ " indicates the mean value, the horizontal bars show the median value, and the top and bottom of the box represent the value of $25^{\text {th }}$ and $75^{\text {th }}$ percentile, respectively. $p$-value was calculated with Student's $t$-test.

d, Proportion of mRNA degradation intermediates of Eif4g2, Ube2d3, Gnas, and Cnbp in mouse liver, heart, and muscle measured by PAIso-seq2. 


\section{Fig. 5 | Proportion of mRNA degradation intermediates indicates the maternal mRNA decay rate during mouse oocyte maturation.}

a, Proportion of mRNA degradation intermediates measured by PAIso-seq 2 and mRNA abundance (relative mRNA copy number) at the transcriptome level from a previous study $^{27}$ in mouse germinal vesicle (GV), meiosis I (MI), and meiosis II (MII) oocytes. Error bars indicate SEM from two replicates.

b, Box plot of the proportion of mRNA degradation intermediates of individual genes in mouse GV, MI, and MII oocytes measured by PAIso-seq2. Genes with at least 20 reads in each sample are included in the analysis $(n=6,517,6,611$, and 6,451 for GV, MI, and MII, respectively).

c, Scatter plot of the proportion of mRNA degradation intermediates of individual genes of samples at neighboring developmental stages in mouse GV, MI, and MII oocytes measured by PAIso-seq2. Each dot represents one gene. Genes with at least 10 reads in each sample are included in the analysis. Number of genes included in the analysis are shown on the top left of each graph.

d, Mathematical model correlating mRNA degradation rate and the proportion of mRNA degradation intermediates for synchronized maturing oocytes. We put together different exponential decay functions with different base numbers, and normalized them with a same initial length $(y=C 1$ when $x=0)$. For function $y 1$ and $y 2$, the function translation along the horizontal axis (- C2) represents the continuous degradation of large amounts of mRNAs. At the test time point (x0), for function y1 (y2), ratio of the length of line segment $\mathrm{BD}(\mathrm{AD})$ to the length of line segment BF (AE) represents the proportion of mRNA degradation intermediates. The positive correlation between mRNA degradation rate and the proportion of mRNA degradation intermediates is shown.

e, Box plot of the proportion of mRNA degradation intermediates of individual genes with different mRNA degradation rate during mouse oocyte maturation measured by PAIso-seq2. The mRNA degradation rate is presented by the ratio of TPM of the current stage to the 

permission.

previous stage. Genes with at least 10 reads in each sample were included in the analysis. Number of genes included in the analysis are shown on the top of each box. $p$-value was calculated with Student's $t$-test.

For all box plots, the " $\times$ " indicates the mean value, the horizontal bars show the median value, and the top and bottom of the box represent the value of $25^{\text {th }}$ and $75^{\text {th }}$ percentile, respectively. 
Fig. 6: Aoroth mRNA, cell, and tissue metabolism in tworisigstems

a Continuous cell cycle progression

GV

(G2 phase of meiosis I)

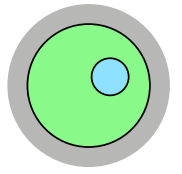

$\mathrm{Ml}$

(Metaphase of meiosis I)
MII

(Metaphase of meiosis II)
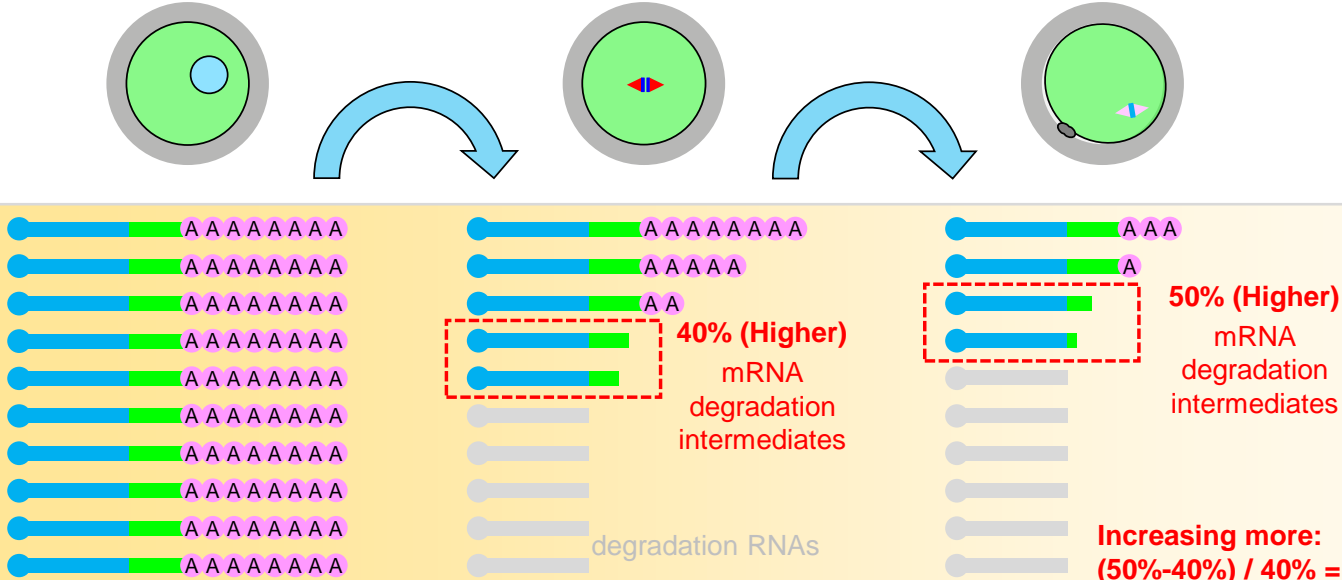

Faster

mRNA metabolism

Increasing more:

(50\%-40\%) / $40 \%=25 \%$
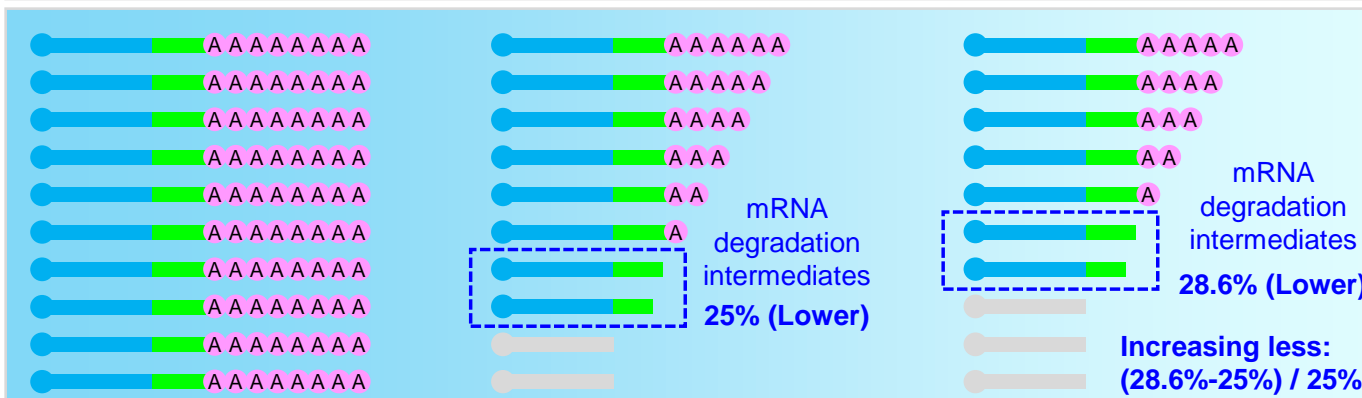

Slower

mRNA metabolism

Increasing less:

$(28.6 \%-25 \%) / 25 \%=14.4 \%$

\section{b Bulk samples}

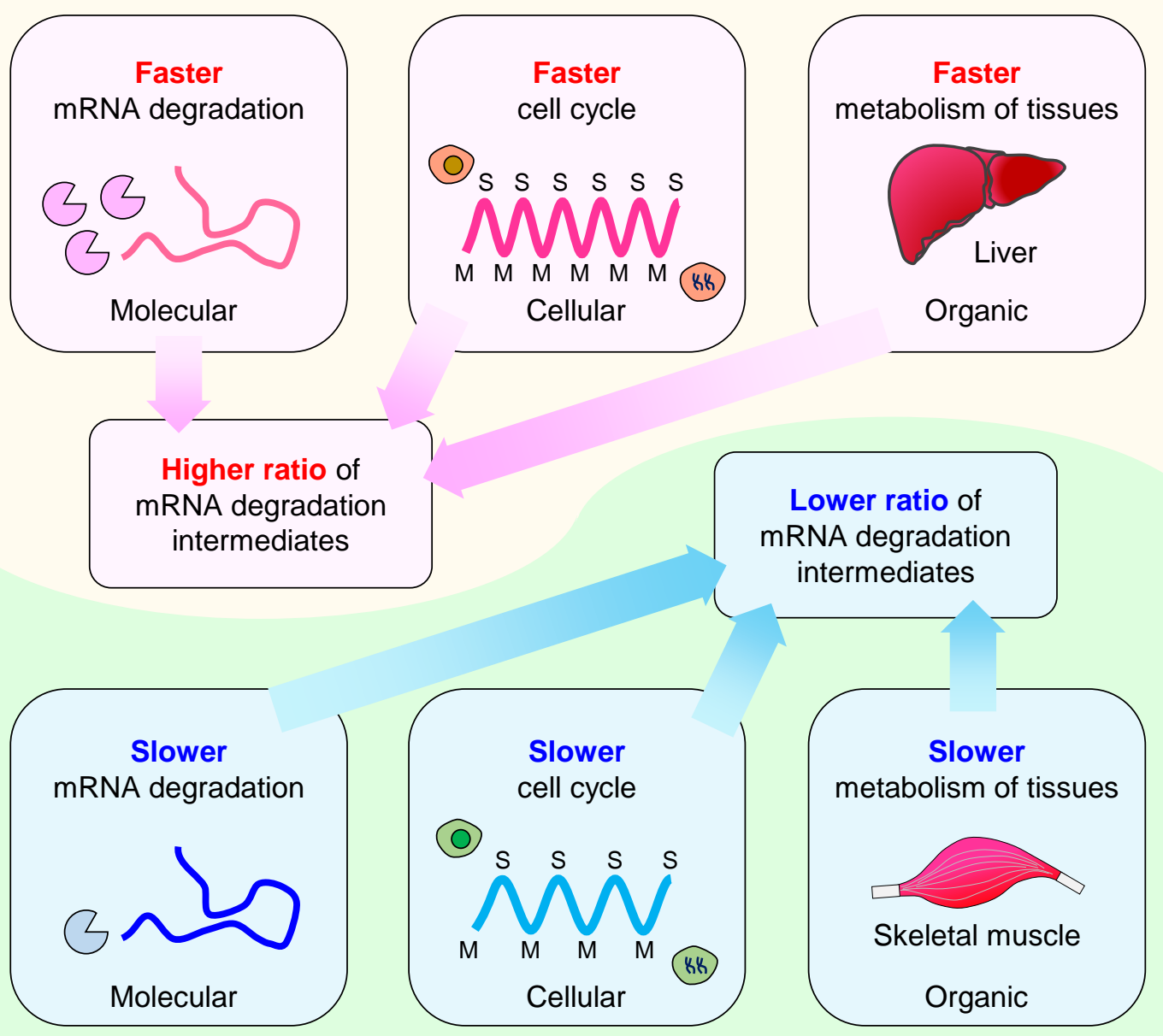


Fig. 6 | A diagram summarizing mRNA degradation intermediates as an indicator of mRNA, cell, and tissue metabolism in two systems.

a, Model showing a positive correlation between the proportion of mRNA degradation intermediates and RNA degradation rate during oocyte maturation.

b, Model showing a positive correlation between the proportion of mRNA degradation intermediates and RNA degradation rate, cell cycle duration, or tissue metabolism rate in bulk samples. 

permission.

\section{Extended Data Fig. 1 | mRNA half-life in 3 T3 and mES cells.}

Box plot of the half-life of mRNA in mES and 3 T3 cells from previously reported data ${ }^{1,7,8,10}$. 